\title{
Reactivity of sulfur centered nucleophiles in photoinduced reactions with 1-bromonaphthalene
}

\author{
Juan E. Argüello, Luciana C. Schmidt, and Alicia B. Peñéñory* \\ Instituto de Investigaciones en Físico Química de Córdoba (INFIQC), Departamento de \\ Química Orgánica, Facultad de Ciencias Químicas, Universidad Nacional de Córdoba, \\ Córdoba (5000), Argentina \\ E-mail: penenory@dqo.fcq.unc.edu.ar
}

\author{
Dedicated to Professor Roberto Arturo Rossi on his 60 $^{\text {th }}$ birthday \\ and to Professor Edmundo A. Rúveda on his $\mathbf{7 0}^{\text {th }}$ birthday \\ (received 06 Sep 03; accepted 10 Oct 03; published on the web 12 Oct 03)
}

\begin{abstract}
The reactivity of $\mathrm{S}^{2-}(\mathbf{1})$, ${ }^{-} \mathrm{SCN}$ (2) and ${ }^{-} \mathrm{SCNH}\left(\mathrm{NH}_{2}\right)$ (3) in photoinduced substitution reactions with 1-bromonaphthalene (5) in DMSO was studied. The photostimulated reaction of ion 1 with 5 renders after quenching with MeI 1-(methylthio)naphthalene (16\%), bis (1-naphthyl) sulfide (17.5\%) and naphthalene (42\%). The lack of reaction in the dark and the inhibition of the photoinduced one by the presence of di-tert-butylnitroxide (DTBN) and $p$-dinitrobenzene ( $p$ DNB) are evidence of a radical chain mechanism for these substitution reactions. On the other hand, ion 2 was unreactive towards 5, both as an electron donor under photostimulation and as a nucleophile. Finally the thiourea anion (3) affords after a photoinduced reaction with 5 and quenching with MeI, 1-methylthionaphthalene (50\%), bis (1-naphthyl) sulfide (22\%) and naphthalene (9\%). This reaction does not occur in the dark and is inhibited in the presence of DTBN. By using hydrogen abstraction from DMSO as competitive reaction, the absolute rate constants for the addition of ions 1, 3 and benzene thiolate ions (4) to 1-naphthyl radicals have been determined to be $0.5 \times 10^{9} \mathrm{M}^{-1} \mathrm{~s}^{-1}, 1.0 \times 10^{9} \mathrm{M}^{-1} \mathrm{~s}^{-1}$ and $5.1 \times 10^{9} \mathrm{M}^{-1} \mathrm{~s}^{-1}$ respectively.
\end{abstract}

Keywords: Thiourea, sodium sulfide, radicals, photoinduced electron transfer, aromatic substitution, reactivity

\section{Introduction}

Radical nucleophilic substitutions involving electron transfer pathways or $\mathrm{S}_{\mathrm{RN}} 1$ is an area of wide interest and its most relevant results have many times been reviewed. ${ }^{1}$ This mechanism has a considerably wide scope in relation to substrates, nucleophiles and synthetic possibilities. 
Sulfur centered nucleophiles, such as thiolate ions have been shown to react with aryl halides under photostimulation to yield a new C-S bond. ${ }^{1 \mathrm{a}, \mathrm{b}}$ While arene thiolate ions (ArS ${ }^{-}$) afford good yields of substitution products, alkane thiolate ions (RS`) yield a mixture of products from fragmentation of the radical anion intermediates and straightforward substitution (scrambling products), making the latter reactions disadvantageous for synthetic purposes. ${ }^{2}$ However, the expected straightforward substitution is mainly obtained with compounds bearing electron withdrawing groups (EWG), polycyclic or heterocyclic halides. ${ }^{3}$

Thioacetate and thiobenzoate ions react with arenediazonium tetrafluoroborates leading to the corresponding aryl thio esters, which can either be isolated or react further, providing a onepot access to other aromatic sulfur derivatives. ${ }^{4}$ The thermal reaction of $\mathrm{Na}_{2} \mathrm{~S}$ with $p$ dichlorobenzene affords the polymer poly( $p$-phenylene sulfide) (PPS). ${ }^{5}$ This reaction is proposed to occur by the ionic $S_{N} A r$ process even though the $S_{R N} 1$ mechanism cannot be totally ruled out. ${ }^{6}$ However, there are no reports on the reactivity of thiocyanate ion (2) towards aryl radicals. On the other hand, the electrochemically initiated reaction of thiourea anion (3) with aryl halides affords moderate yields of a mixture of $\mathrm{ArSH}, \mathrm{ArSSAr}$ and $\mathrm{ArSAr}$, ascribed to the fragmentation of the radical anion intermediates (eq. 1). ${ }^{7}$

$$
\begin{aligned}
& \mathrm{ArX}+{ }_{\mathrm{HN}} \underset{3}{\stackrel{\mathrm{S}^{-}}{\mathrm{NH}_{2}}} \frac{\begin{array}{c}
\text { electrode } \\
\text { redox mediator }
\end{array}}{\mathrm{NH}_{3}} \mathrm{ArSH}+\mathrm{Ar}_{2} \mathrm{~S}+\mathrm{Ar}_{2} \mathrm{~S}_{2} \\
& \mathrm{ArX}=4-\mathrm{NCC}_{6} \mathrm{H}_{4} \mathrm{Cl} \text { and 2-, 3-, 4-chloropyridines }
\end{aligned}
$$

In addition, the photochemical aromatic substitution of aryl halides by thiourea was also described. ${ }^{8}$ This reaction proceeds under UV irradiation with $25-76 \%$ yields of ArSSAr, for aryl derivatives bearing electron donating substituents such as $\mathrm{NH}_{2}, \mathrm{NR}_{2}$ and OMe. However, 1bromonaphthalene was unreactive under these reaction conditions. ${ }^{8}$

We have previously studied the reactivity of thiourea anion (3) in photoinduced aromatic radical nucleophilic substitution as a "one pot” method for the synthesis of aryl thiols, alkyl aryl sulfides, symmetrical or unsymmetrical diaryl sulfides and diaryl disulfides from moderate to good yields. ${ }^{9}$ These results have encouraged us to further explore the reactivity and the potential of related sulfur anions for the synthesis of aromatic sulfur compounds. Thus, we report herein the photoinduced reaction of $\mathrm{S}^{2-}(\mathbf{1})$ and ${ }^{-S C N}$ (2) with haloarenes in DMSO and the absolute rate constants for the coupling reactions of ions $\mathbf{1}, \mathbf{3}$ in competitive reactions with benzene thiolate ion (4) towards 1-naphthyl radicals.

\section{Results and Discussion}

Table 1 summarizes the results obtained with the nucleophiles $\mathbf{1}, 2$ and $\mathbf{3}$ in their photoinduced reactions with 1-bromonaphthalene (5). 
Table 1. Reactions of sulfur nucleophiles with 1-bromonaphthalene (5) in $\mathrm{DMSO}^{a}$

\begin{tabular}{lccccccc}
\hline Entry & "S"- & Cond. & Comp. added & \multicolumn{4}{c}{ Products yield (\%) } \\
& & Time (h) & & Convn & ArH & ArSMe & ArSAr \\
\hline 1 & $\mathbf{1}^{c}$ & $3, \mathrm{~h} v$ & & 100 & 42 & $16^{d}$ & 17.5 \\
$2^{e}$ & & $3, \mathrm{~h} v$ & $10 \% \mathrm{~V} / \mathrm{V} \mathrm{H}_{2} \mathrm{O}$ & 83 & 67 & 3 & 11 \\
3 & & 3, dark & & 0 & - & - & - \\
4 & & $1, \mathrm{~h} v$ & & 97 & 46 & $16^{d}$ & 16 \\
5 & & $0.5, \mathrm{~h} v$ & & 63 & 30 & 9 & 13 \\
6 & & $0.5, \mathrm{~h} v$ & $0.01 \mathrm{M}$ DTBN & 51 & 25 & 8 & 10 \\
7 & & $0.5, \mathrm{~h} v$ & $0.01 \mathrm{M} p$-DNB & 12 & 3.4 & 1.3 & 1 \\
8 & $\mathbf{1}^{e}$ & $3, \mathrm{~h} v$ & & 100 & 40 & $18^{d}$ & 34 \\
$9^{f}$ & $\mathbf{2}$ & $3, \mathrm{~h} v$ & & 17 & 4.4 & - & - \\
$10^{f}$ & & $3, \mathrm{~h} v$ & $t$-BuOK (2 eq.) & 47 & 23 & - & - \\
$11^{f}$ & & $3, \mathrm{~h} v$ & $\mathbf{1}^{c}$ (5 eq.) & 100 & 52 & 12 & 16 \\
$12^{g}$ & $\mathbf{3}$ & $3, \mathrm{~h} v$ & & 100 & 13 & $37^{d}$ & 32 \\
$13^{f, g}$ & & $3, \mathrm{~h} v$ & & 100 & 9 & 50 & 22 \\
$14^{f, g}$ & & $1, \mathrm{~h} v$ & & 100 & 11 & 54 & 24 \\
$15^{f, g}$ & & $0.25, \mathrm{~h} v$ & & 91 & 9 & 48 & 19 \\
\hline
\end{tabular}

${ }^{a} \mathrm{ArX}=1-\mathrm{BrC}_{10} \mathrm{H}_{7}$ : 0.05M, "S"- sulfur centered nucleophiles: $0.25 \mathrm{M}$. ${ }^{b}$ Determined by GC using the internal standard method, error $5 \%$. The conversion was determined by quantification of the recovered substrate. ${ }^{c} \mathrm{Na}_{2} \mathrm{~S} .9 \mathrm{H}_{2} \mathrm{O}$. ${ }^{d}$ Together with 2-3\% of the 2-(methylthio) naphthalene from the 2-bromonaphthalene present as impurity of the bromide $5 .{ }^{e} \mathrm{Na}_{2} \mathrm{~S}$ anhydrous. ${ }^{f}$ Nucleophile: 1 $\mathrm{BrC}_{10} \mathrm{H}_{7}$ ratio of 10:1. ${ }^{g}$ From reference 9.

The hydrated salt of $\mathrm{Na}_{2} \mathrm{~S}^{10}$ was partially soluble in DMSO, and after $3 \mathrm{~h}$ of irradiation with 5, this reaction affored a mixture of naphthalene, 1- (methylthio)naphthalene and bis (1naphthyl) sulfide, after quenching with MeI. The addition of water improves the solubility of $\mathrm{Na}_{2} \mathrm{~S}$, but decreases the reactivity and enhances the amount of the reduction product naphthalene. The photoinduced reaction is completed in $1 \mathrm{~h}$ and proceeds to a 63\% conversion in half hour. There was no reaction in the dark after $3 \mathrm{~h}$ and the photoinduced reaction carried out in $0.5 \mathrm{~h}$ was slightly inhibited by the presence of di-tert-butylnitroxide (DTBN), and strongly suppressed by $p$-dinitrobenzene ( $p$-DNB) (Table 1, entries 1-7). These results and the presence of an important amount of naphthalene, can be ascribed to a radical chain substitution mechanism of substitution $\left(\mathrm{S}_{\mathrm{RN}} 1\right)$, involving an electron transfer process from the ion $\mathbf{1}$ to the naphthyl bromide as the initiation step (eq. 2). Subsequent fragmentation of the radical anion thus formed, affords the 1naphthyl radical, which by coupling with anion 1 yields a new radical anion 6 (eq. 3). Electron transfer to the halide 5 renders the 1-naphthalene thiolate ion (7) and the radical anion of the bromide 5, which continues the chain propagation cycle (eqs. 3 and 4). Further reaction of ion 7 with 1-naphthyl radical followed by electron transfer to $\mathbf{5}$, finally yields the disubstitution 
product bis (1-naphthyl) sulfide (eq. 5). Hydrogen atom abstraction from the solvent by 1naphthyl radicals, gives the reduction product naphthalene (eq. 6). After irradiation, the reaction mixture is quenched by MeI to afford 1-(methylthio)naphthalene (eq. 7).

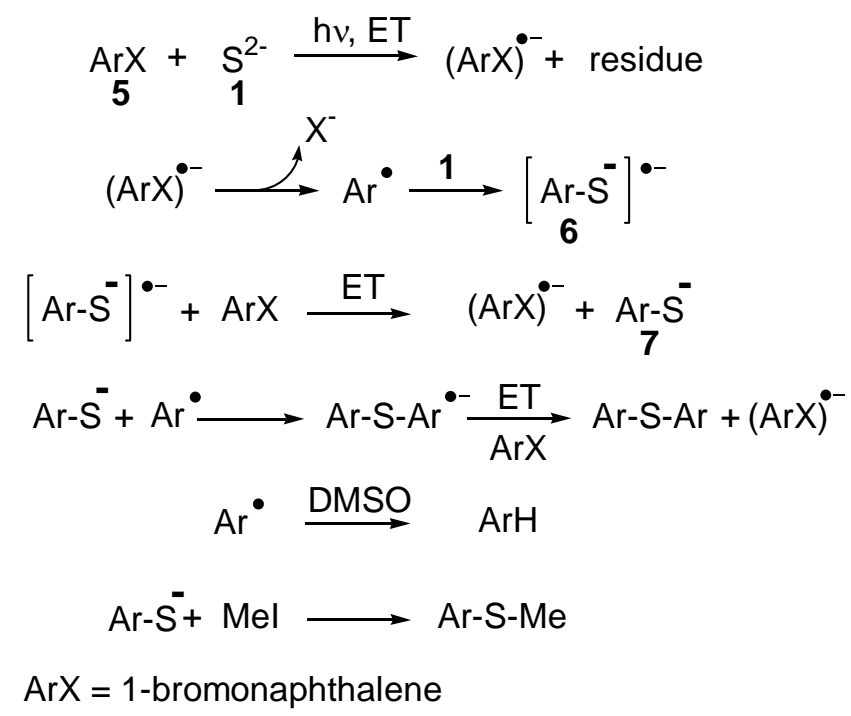

As previously described, $\mathrm{Na}_{2} \mathrm{~S}$ formed by reaction of sulfur and sodium metal in liquid ammonia, did not react with iodobenzene after $4 \mathrm{~h}$ of irradiation. ${ }^{11}$ We now report that ion $\mathbf{1}$ is able to add to the more electrophilic 1-naphthyl radicals. To the best of our knowledge, this is the first report that clearly showed that $\mathrm{Na}_{2} \mathrm{~S}$ is able to react by the $\mathrm{S}_{\mathrm{RN}} 1$ mechanism under photostimulation. When the reaction was performed with anhydrous $\mathrm{Na}_{2} \mathrm{~S}$, although the naphthalene produced was practically the same, the product ratio substitution/reduction increased from 0.7 to 1.3 (Table 1, entry 8). This ratio can not be improved by increasing the concentration of the nucleophile due to the insolubility of the $\mathrm{Na}_{2} \mathrm{~S}$ salt in DMSO at higher concentration than $0.25 \mathrm{M}$. The photoinduced reaction of ion $\mathbf{1}$ with the electrophilic 4-benzoylphenyl radical yielded 4-(methylthio)benzophenone (54\%), bis (4-benzoylphenyl) sulfide (24\%) and benzophenone (7\%). ${ }^{12}$

The ${ }^{-} \mathrm{SCN}$ ion (2) was unreactive both in the initiation step as electron donor and in the addition to 1-naphthyl radicals generated by a photostimulated entrainment reaction with tert$\mathrm{BuOK}$ or $\mathrm{Na}_{2} \mathrm{~S}$. Although this anion would be able to add to the radical, a very fast dissociation of the radical anion intermediate into aryl thiyl radical and cyanide anion would prevent to built up a propagation cycle (Table 1, entries 9-11). A similar behavior has been previously observed with 2-bromo-2-nitropropane. ${ }^{13}$ Thiocyanate anion failed to react with this activated bromide by an $\mathrm{S}_{\mathrm{RN}} 1$ mechanism, even under entrainment conditions with $\mathrm{Me}_{2} \mathrm{CNO}_{2}{ }^{-}$. This anion adds easily to the 2-nitro-2-propyl radical (under oxidative conditions with Fe III), but the radical anion dissociates rapidly, as showed by ESR spectroscopic studies, suggesting that dissociation is faster than ET to the substrate, inhibiting the propagation cycle of the $\mathrm{S}_{\mathrm{RN}} 1$ reaction. ${ }^{13}$ 
The thiourea anion (3) affords, after a photoinduced reaction with bromide 5 and quenching with MeI, 1-(methylthio)naphthalene (37\%), bis(1-naphthyl) sulfide (32\%) and naphthalene (13\%). When the reaction was performed with a ratio nucleophile/substrate of 10 , the substitution products increased, and after only fifteen minutes the conversion was $91 \%$. This reaction is strongly inhibited by DTBN and there is no conversion at all in the dark (Table 1 , entries 12-15). Based on these results, an $\mathrm{S}_{\mathrm{RN}} 1$ mechanism is assumed for these reactions and the arene thiolate ion $\mathbf{7}$ and radical $\mathbf{9}$ are formed by addition of ion $\mathbf{3}$ to the aryl radical followed by fragmentation of the radical anion intermediate $\mathbf{8}$, (eq. 8). ${ }^{9}$

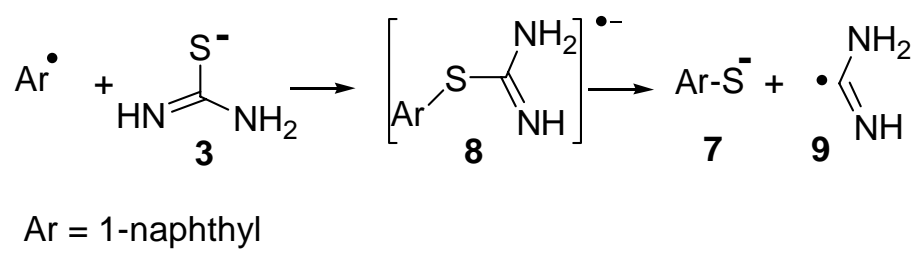

The independent experiments 8 and 12 in Table 1, clearly reveal that ion $\mathbf{3}$ is more reactive than ion 1. Taking into account that the $\mathrm{S}_{\mathrm{RN}} 1$ process comprises initiation, propagation, and termination steps, it was of interest to evaluate the relative reactivity of ions $\mathbf{1}$ and $\mathbf{3}$ toward 1naphthyl radicals in competitive experiments. Since both nucleophiles render the same product, namely 1-naphthalene thiolate ion (7), we choose benzene thiolate ion (4) as partner for carried out separated competitive reactions, and to avoid the further competition for 1-naphthyl radicals by ion 7 as indicated in equation 5 . Furthermore, the absolute rate constants for the coupling $\left(k_{\mathrm{c}}\right)$ of 1-naphthyl radicals with the nucleophiles $\mathbf{1}, \mathbf{3}$ and $\mathbf{4}$ can be determined in DMSO using hydrogen abstraction from the solvent as competitive reaction, whose rate constant $k_{\mathrm{H}}$ has been determined to be $7.1 \times 10^{6} \mathrm{M}^{-1} \mathrm{~s}^{-1}$ at $25^{\circ} \mathrm{C}^{14}$. Three competitive steps are possible for the intermediate aryl radicals as outlined in Scheme 1: step (a) coupling with the ion $\mathbf{1}$ or $\mathbf{3}$ to yield after electron transfer or fragmentation, the substitution product 1-naphthalene thiolate ion (7); step (b) hydrogen abstraction from the solvent DMSO to render the reduction product naphthalene; and step (c) coupling with the benzene thiolate ion (4) to afford the substitution product (1-naphthyl) phenyl sulfide after electron transfer.

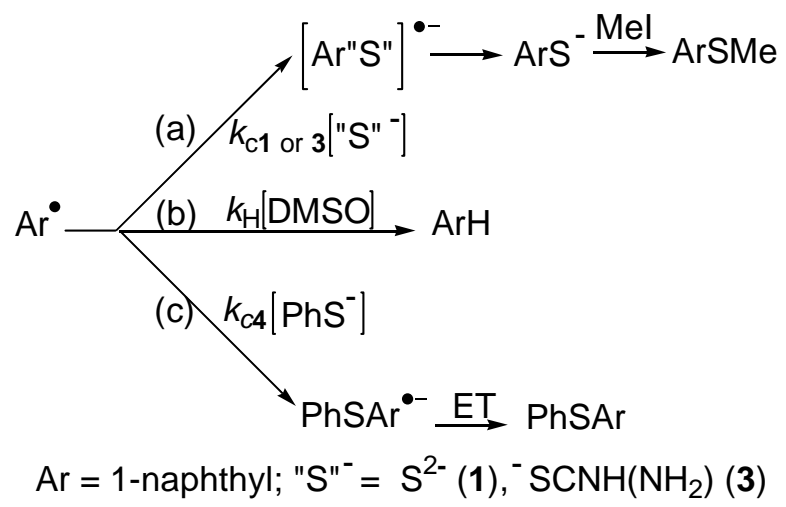

\section{Scheme 1}


Once the yields from the substitutions (ArSMe and PhSAr) and reduction (ArH) products were determined in the photoinduced reactions of 1-bromonaphthalene with ions $\mathbf{1}, \mathbf{3}$ and $\mathbf{4}$ in excess, it was possible to calculate the $k_{\mathrm{c}}$ by using equation 9 and the relative reactivities by using equation 10, (Table 2). ${ }^{15}$ For the ion $\mathbf{4}$ we obtained from the competitive experiments with 1 or 3, two different values for $k_{\mathrm{c} 4}$, that is $2.2 \times 10^{9} \mathrm{M}^{-1} \mathrm{~s}^{-1}$ or $5.1 \times 10^{9} \mathrm{M}^{-1} \mathrm{~s}^{-1}$ respectively. Taking into account that the presence of water in the $\mathrm{Na}_{2} \mathrm{~S}$ salt decreased the product ratio substitution/reduction (Table1, entries 1,2 and 8) and that $\mathrm{Na}_{2} \mathrm{~S}$ is highly hygroscopic, we consider the latter value more reliable. Making the same correction for ion 1 , a $k_{\mathrm{c} 1}=0.5 \times 10^{9} \mathrm{M}^{-}$ ${ }^{1} \mathrm{~s}^{-1}$ is obtained. The rate constant measured for thiourea ion is comparable to the value obtained in liquid ammonia toward 4-cyanophenyl radical $\left(4.2 \times 10^{9} \mathrm{M}^{-1} \mathrm{~s}^{-1}\right){ }^{7}$ Taking ion $\mathbf{1}$ as reference, the following reactivity order is obtained: $\mathrm{S}^{2-}(\mathbf{1})(1.00)$, ${ }^{-} \mathrm{SCNH}\left(\mathrm{NH}_{2}\right)$ (3) (2) and $\mathrm{PhS}^{-}$(4) (11).

$$
\begin{aligned}
& \frac{k_{\mathrm{C}}}{k_{\mathrm{H}}}=\frac{[\mathrm{ArNu}]_{t}[\mathrm{DMSO}]}{[\mathrm{ArH}]_{t}[\mathrm{Nu}]_{o}} \\
& \frac{k_{\mathrm{c} 4}}{k_{\mathrm{c} \text { or } 3}}=\frac{[\mathrm{ArSPh}]_{t}\left[\mathrm{HS}^{-}\right]_{o}}{[\mathrm{ArSMe}]_{t}[4]_{o}}
\end{aligned}
$$

With a similar strategy Galli and coworkers have measured the rate constant for the addition of the pinacolone enolate ion to 9-anthracenyl and 1-naphthyl radicals $\left(4.4 \times 10^{8}\right.$ and $2.9 \times 10^{9}$ $\mathrm{M}^{-1} \mathrm{~s}^{-1}$, respectively) ${ }^{16 \mathrm{a}}$ and more recently, the coupling of various nucleophiles to vinyl radicals was investigated. ${ }^{16 \mathrm{~b}}$ In general the additions of nucleophiles to aryl radicals, other than the simplest phenyl radicals, are very fast reactions and range from $10^{8}-10^{10} \mathrm{M}^{-1} \mathrm{~s}^{-1}$.

Table 2. Rate constant $\left(k_{\mathrm{c}}\right)$ for the reaction of 1-naphthyl radical with sulfur nucleophiles in

\begin{tabular}{|c|c|c|c|c|c|c|c|c|c|}
\hline \multirow[t]{2}{*}{ Entry } & \multirow{2}{*}{$\begin{array}{l}\text { "S"- } \\
{[\mathrm{M}]}\end{array}$} & \multirow{2}{*}{$\begin{array}{c}\mathbf{4} \\
{[\mathrm{M}]}\end{array}$} & \multirow{2}{*}{$\begin{array}{l}\text { Time } \\
\text { [min] }\end{array}$} & \multicolumn{3}{|c|}{ Products yield (\%) } & \multirow[t]{2}{*}{$\mathrm{k}_{\mathrm{c} 4} / \mathrm{k}_{\mathrm{c} 1 \text { or } 3}$} & \multirow{2}{*}{$\begin{array}{c}\mathrm{k}_{\mathrm{c} 4}{ }^{-1} \\
{\left[\mathrm{M}^{-1} \mathrm{~s}^{-1}\right]}\end{array}$} & \multirow{2}{*}{$\begin{array}{c}\mathrm{k}_{\mathrm{c}}^{b} \\
{\left[\mathrm{M}^{-1} \mathrm{~s}^{-1}\right]}\end{array}$} \\
\hline & & & & ArSMe & $\mathrm{ArSPh}$ & $\mathrm{ArH}$ & & & \\
\hline 1 & $1,0.25$ & 0.125 & 120 & 8.6 & 480 & 160 & 11 & $2.2 \times 10^{9}$ & $0.2 \times 10^{9}$ \\
\hline 2 & $3,0.50$ & 0.250 & 60 & 20.4 & 51.0 & 4.0 & 5 & $5.1 \times 10^{9}$ & $1.0 \times 10^{9}$ \\
\hline
\end{tabular}
DMSO at $25^{\circ} \mathrm{C}^{a}$

${ }^{a}$ Photostimulation at $300 \mathrm{~nm}$ in $10 \mathrm{~mL}$ of DMSO. [1- $\left.\mathrm{BrC}_{10} \mathrm{H}_{7}\right]=0.05 \mathrm{M}$. Products yield determined by GC using the internal standard method. ${ }^{b}$ From duplicated experiments: average error $\leq 10 \%$.

In summary, we report for the first time the photoinduced radical chain nucleophilic substitutions of aryl halides by sulfide ions. The relative reactivities of sulfide, thiourea and benzene thiolate ions towards 1-naphthyl radicals were measured, and from the least to the most reactive nucleophile, the span in reactivity is $\sim 1$ order of magnitude. The absolute rate constant measured range from $0.5 \times 10^{9}-5.1 \times 10^{9} \mathrm{M}^{-1} \mathrm{~s}^{-1}$. These results are consistent with the concept that the coupling of nucleophiles with aryl radicals occurs at near the encounter controlled limit. ${ }^{1}$ 
Alkyl aryl sulfides can be achieved by quenching the reaction mixture with an adequate alkyl halide (methyl iodide in the present report). Further transformations of the "in situ" generated arene thiolate ions are possible, such as protonation to the thiols, oxidation to the diaryl disulfides, subsequent $\mathrm{S}_{\mathrm{RN}} 1$ reaction to afford unsymmetrical diaryl sulfide, etc. Work is in progress to study the scope and limitations of this strategy for the syntheses of sulfur aromatic compounds.

\section{Experimental Section}

General Procedures. The general methods and procedures for the photoinduced reaction are the same as published before. ${ }^{17}{ }^{1} \mathrm{H}$ and ${ }^{13} \mathrm{C}$ NMR spectra were recorded at 200 and $50 \mathrm{MHz}$ respectively on a Brucker AC-200 spectrometer, and all spectra are reported in $\delta(\mathrm{ppm})$ relative to $\mathrm{Me}_{4} \mathrm{Si}$, with $\mathrm{CDCl}_{3}$ as solvent. Gas chromatographic analyses were performed on a Hewlett Packard 6890 A with a flame-ionization detector, on a HP-5 30 m capillary column of a $0.32 \mathrm{~mm}$ x $0.25 \mu \mathrm{m}$ film thickness, or a HP1 $5 \mathrm{~m}$ x 0.53 x $2.65 \mu \mathrm{m}$ film thickness column. GS/MS analyses were carried out on a Shimadzu GC-MS QP 5050 spectrometer, employing a 25 m x 0.2 mm x $0.33 \mu \mathrm{m}$ HP-5 column.

Materials. $t$-BuOK, thiourea, $\mathrm{KSCN}, \mathrm{Na}_{2} \mathrm{~S} .9 \mathrm{H}_{2} \mathrm{O}$, 1-bromonaphthalene (contains <3\% 2bromonaphthalene), 4-bromobenzophenone, naphthalene, benzophenone, thiophenol, $p$ dinitrobenzene and DTBN were all high purity commercial samples which were used without further purification. The commercially available $\mathrm{Na}_{2} \mathrm{~S} .9 \mathrm{H}_{2} \mathrm{O}$ was dehydrated according to literature. ${ }^{18}$ DMSO was distilled under vacuum and stored over molecular sieves (4 $\AA$ ). The anions 3 and $\mathbf{4}\left(\mathrm{PhS}^{-}\right)$were generated in situ by acid-base deprotonation using tert-BuOK.

All the products are known and exhibited physical properties identical to those reported in the literature. Also, they were isolated by radial chromatography from the reaction mixture and characterized by ${ }^{1} \mathrm{H}$ and ${ }^{13} \mathrm{C}$ NMR and mass spectrometry.

Registry No.: 1-(methylthio)naphthalene, ${ }^{19}$ [10075-72-6]; 2-(methylthio)naphthalene, ${ }^{20}$ [7433-79-6]; bis (1-naphthyl) sulfide, ${ }^{21}$ [607-53-4]; 4-(methylthio)benzophenone, ${ }^{22}$ [23405-483]; 1-naphthyl phenyl sulfide, ${ }^{23}$ [7570-98-1]; and bis (4-benzoylphenyl) sulfide, ${ }^{24}$ [47632-47-3].

Photoinduced reactions of anion 1 with 5 general procedure. The photochemical reaction was carried out in a three-necked, $10 \mathrm{~mL}$ Schlenk tube equipped with a nitrogen gas inlet and a magnetic stirrer. The flask was dried under vacuum, filled with nitrogen and then charged with $10 \mathrm{~mL}$ of dried DMSO. Then $2.5 \mathrm{mmol}$ of the nucleophile 1 and $0.5 \mathrm{mmol}$ of 1 -

bromonaphthalene (5) were added to the degassed solvent under nitrogen. After $3 \mathrm{~h}$ of irradiation with a medium pressure Hg lamp, the reaction was quenched by the addition of MeI (6 equiv) in excess and $30 \mathrm{~mL}$ of water, and then the mixture was extracted with methylene chloride 
(3x20mL). The organic extract was washed twice with water, dried and the products were quantified by GC with diphenyl disulfide as internal standard.

Determination of the rate constant $\boldsymbol{k}_{\mathrm{c}}$ for the reaction of 1-naphthyl radical with ions $\mathbf{1 ,} 3$ and 4. The reaction of ions 1 and 4 with 5 is representative: $2.5 \mathrm{mmol}$ of salt $1,1.25 \mathrm{mmol}$ of benzene thiol and $1.1 \mathrm{mmol}$ of tert-BuOK were added to $10 \mathrm{~mL}$ of dried and degassed DMSO in a $10 \mathrm{~mL}$ Schlenk tube, under a nitrogen atmosphere. To the solution of nucleophiles 1 and 4 , was added $0.5 \mathrm{mmol}$ of 5 and the solution was irradiated for $60 \mathrm{~min}$ at $25^{\circ} \mathrm{C}$. After quenching with MeI, the mixture was extracted with methylene chloride $(3 x 20 \mathrm{~mL})$. The organic extract was washed twice with water, dried and the GC yields for the reduction and substitution products were determined by the internal standard method. Equation 9 allowed for the calculation of the $k_{\mathrm{c}}$ using 14.1 $\mathrm{M}$ for the concentration of DMSO.

\section{Acknowledgements}

This work was supported partly by the Consejo Nacional de Investigaciones Científicas y Técnicas (CONICET) and FONCYT, Argentina, the Alexander von Humboldt Foundation and the Third World Academic of Sciences (TWAS). J. E. A. gratefully acknowledges the receipt of a fellowship from CONICET.

\section{References}

1. (a) Rossi, R. A.; Pierini, A. B.; Peñéñory, A. B. Chem. Rev. 2003, 103, 71. (b) Rossi, R. A.; Pierini, A. B.; Santiago, A. N. In Organic Reactions. Paquette, L. A.; Bittman, R. Eds.; John Wiley \& Sons, Inc. 1999; pp 1-271. (c) Rossi, R. A.; Peñéñory, A. B In Handbok of Organic Photochemistry and Photobiology, $2^{\text {nd }}$ Edn., Horspool, W. M., Ed.; CRC Press Inc. LLC: Boca Raton, 2003, Ch. 47, in press. (d) Savéant, J. M. Acc. Chem. Res. 1993, 26, 455. (e) Savéant, J. M. Adv. Phys. Org. Chem. 1990, 26, 1.

2. Rossi, R. A.; Palacios, S. M. J. Org. Chem. 1981, 46, 5300.

3. Beugelmans, R.; Bois-Choussy, M.; Boudet, B. Tetrahedron 1983, 39, 4153.

4. Petrillo, G.; Novi, M.; Garbarino, G.; Filiberti, M. Tetrahedron 1989, 45, 7411.

5. (a) Annenkova, V. Z.; Antonik, L. M.; Shafeeva, I. V.; Vakul'skaya, T. I.; Vitkovskii, V. Yu; Voronkov, M. G. Vysokomol. Soed., Ser.B 1986, 28, 137; Chem. Abstr. 1986, 105, 6848r. (b) Annenkova, V. Z.; Antonik, L. M.; Vakul'skaya, T. I.; Voronkov, M. G. Dokl. Akad. Nauk SSSR 1986, 286, 1400; Chem. Abstr. 1986, 105, 190409x.

6. Fahey, D. R.; Ash, C. E. Macromolecules 1991, 24, 4242.

7. Combellas, C.; Dellerue, S.; Mathey, G.; Thiébault, A. Tetrahedron Lett. 1997, 38, 539. 
8. Frolov, A. N.; Klokova, E. M.; El ' tsov, A. V. J. Org. Chem. USSR, (Engl. Transl.) 1981, 17, 1926.

9. Argüello, J. E., Schmidt, L. C.; Peñéñory, A. B. Org. Lett. 2003, in press.

10. The commercial $\mathrm{Na}_{2} \mathrm{~S} .9 \mathrm{H}_{2} \mathrm{O}$ was used.

11. Rossi, R. A.; Peñéñory, A. B. J. Org. Chem. 1981, 46, 4580.

12. Reaction performed with 4-bromobenzophenone $0.05 \mathrm{M}$ and $\mathrm{S}^{2-} 0.25 \mathrm{M}$. After $3 \mathrm{~h}$ of irradiation with a medium pressure $\mathrm{Hg}$ lamp, the reaction was quenched by the addition of MeI (6 equiv) in excess, and quantified by GC using the internal standard method.

13. AlKhalil, S. I.; Bowman, W. R.; Gaitonde, K.; Marley, M. A.; Richardson, G. D. J. Chem. Soc. Perkin Trans. 2 2001, 1557.

14. Andrieux, C. P.; Savéant, J.-M.; Su, K. B. J. Phys. Chem. 1986, 90, 3815.

15. $\left[\mathrm{Nu}^{-}\right]_{\mathrm{o}}$ is the initial concentration of the nucleophile; $\mathrm{ArH}$ and $\mathrm{ArNu}$ are the concentrations of the reduction and substitution products at time $t$. These equations are based on the assumption that the reactions of the aryl radicals with the nucleophiles and the solvent are first order in the latter species, and their concentrations are constant during the experiments.

16. (a) Annunziata, A.; Galli, C.; Marinelli, M.; Pau, T. Eur. J. Org. Chem. 2001, 1323. (b) Branchi, B.; Galli, C.; Gentili, P. Eur. J. Org. Chem. 2002, 2844.

17. Argüello, J. E.; Peñéñory, A. B.; Rossi, R. A. J. Org. Chem. 2000, 65, 7175.

18. Coutois, G. C. R. hebd. Séances Acad. Sci. 1938, 207, 1220.

19. Gilman, H.; Webb, F. J. J. Am. Chem. Soc. 1949, 71, 4062.

20. Buu-Hoi, Ng. Ph.; Hoán, Ng.; Lavit, D. J. Chem. Soc. 1953, 485. Jacques, J. Bull. Soc. Chim. France 1955, 231.

21. Hauptmann, H.; Wladislaw, B. J. Am. Chem. Soc. 1950, 72, 710.

22. Mustafá, A. J. Chem. Soc. 1949, 352.

23. Bunnett, J. F.; Creary, X. J. Org. Chem. 1974, 39, 3173.

24. Guggenheim, T. L., Tetrahedron Lett. 1987, 28, 6139. 